\title{
CARACTERÍSTICAS FÍSICO-QUÍMICAS DE PHYSALIS EM DIFERENTES COLORAÇÕES DO CÁLICE E SISTEMAS DE CONDUÇÃO ${ }^{1}$
}

\author{
CLÁUDIA SIMONE MADRUGA LIMA² JOSEANA SEVERO ${ }^{3}$, ROBERTA MANICA-BERTO ${ }^{4}$, \\ JORGE ADOLFO SILVA ${ }^{5}$, LEO RUFATO ${ }^{6}$, ANDREA DE ROSSI RUFATO $^{7}$
}

RESUMO -Uma espécie que está sendo incorporada no cultivo de pequenas frutas é a physalis. Esta Solanaceae é considerada uma planta rústica que tem dificuldade de manter suas hastes eretas, seus frutos estão dentro de um cálice que os protege contra insetos, pássaros e condições adversas. O objetivo neste trabalho foi avaliar as características físico-químicas dos frutos de Physalis peruviana em função das diferentes colorações de cálice e dos sistemas de condução utilizados. A colheita dos frutos foi realizada levando em consideração cinco diferentes classificações quanto à coloração de cálice: 1 (verde); 2 (verde-amarelada); 3 (amarelo-esverdeada); 4 (amarela), e 5 (amarelo-amarronzada), provenientes dos sistemas de condução "V" invertido e triangular. O delineamento experimental foi o inteiramente casualizado, com esquema fatorial $5 \times 2$ (colorações do cálice x sistemas de condução). A unidade experimental foi composta de dez frutos, sendo cada tratamento repetido três vezes. Os dados foram submetidos à análise de variância (ANOVA), aplicando o teste de Tukey, ao nível de 5\% de probabilidade de erro, para comparação das médias. As variáveis avaliadas foram: massa fresca do fruto, do cálice e total, diâmetro, firmeza, coloração da epiderme, sólidos solúveis totais (SST), acidez total titulável (ATT), razão SST/ATT e pH. O sistema de condução triangular, combinado às colorações de fruto amarela e amarelo-amarronzada resultou, respectivamente, em frutos com maior conteúdo de SST e razão SST/ATT. Os maiores valores para massa e diâmetro de fruto foram alcançados quando os cálices se apresentavam amarelo-amarronzados, enquanto a maior massa total foi atingida quando os cálices tinham coloração amarela. Já para a massa do cálice, as maiores respostas foram obtidas nos de coloração verde. Conclui-se que physalis colhidos a partir da terceira fase (3) (cálice amarelo-esverdeado) apresentam o melhor conjunto de características físico-químicas, em ambos os sistemas de condução avaliados.

Termos para indexação: Physalis peruviana, frutos, colheita, sistema "V" invertido, sistema triangular.

\section{CHEMICAL CHARACTERISTICS OF CAPE-GOOSEBERRY FRUITS IN DIFFERENT SEPAL COLORS AND TRAINING SYSTEMS}

\begin{abstract}
A species that is being introduced in the plantations of small fruits is the cape-gooseberry. The Solanaceae is considered a rugged plant that is difficult to maintain its upright stems; its fruits are in a glass that protects against insects, birds, and adverse conditions. This objective of this work was to evaluate the physicochemical characteristics of Physalis peruviana fruits according to different sepal colors and the used training systems. The fruits were harvested and classified in five different sepal color groups: 1 (green), 2 (green-yellowish), 3 (yellow-greenish), 4 (yellow), 5 (yellow-brownish); and two training system groups: inverted " $\mathrm{V}$ " and triangular. The experimental design was completely randomized in a factorial scheme $5 \times 2$ (sepal colors $\mathrm{x}$ drive systems). The experimental unit was composed of ten fruits, with each treatment repeated three times. The data were submitted to variance analysis (ANOVA) by applying the Tukey test at a $5 \%$ level of probability of error for the comparison of the means. The appraised variables were: fruit, sepals and total fresh mass, diameter, firmness, epidermis color, total soluble solids (TSS), total titratable acidity (TTA), the TSS/TTA ratio and $\mathrm{pH}$. The triangular training system associated with yellow and yellowbrownish sepals resulted, respectively, in fruits with more SST and better TSS/TTA ratio. The highest mass and diameter values were obtained in cape-gooseberry fruits with yellow-brownish sepals, while the highest total mass was reached when sepals had yellow color. For sepals mass, the largest responses were obtained with the green sepals. From this physicochemical characterization we can conclude that cape-gooseberry picked $\mathrm{g}$ from the third color phase (yellow-greenish) present the best physiochemical characteristics in both assessed training systems.
\end{abstract}

Index terms: Physalis peruviana, fruit, harvest, triangle system, inverted "V" system.

${ }^{1}$ (Trabalho 248-08). Recebido em:29-09-2008. Aceito para publicação em: 23-06-2009. Apoio financeiro CAPES ${ }^{2}$ Enga Agr., mestranda em Fruticultura, UFPel, Depto de Fitotecnia, Pelotas-RS. . E-mail: claudialim@pop.com.br

${ }^{3}$ Química Industrial de Alimentos, mestranda em Ciência e Tecnologia Agroindustrial, UFpel, Depto. de Ciência e Tecnologia Agroindustrial. Pelotas-RS. E-mail: josi_severo@yahoo.com.br

${ }^{4} \mathrm{Eng}^{\mathrm{a}}$ Agr., Msc. Doutoranda em Fruticultura, UFpel, Depto de Fitotecnia.Pelotas-RS. E-mail: robertamanica@yahoo.com.br ${ }^{5}$ Eng .Agr., Dr. Prof. do Depto. de Ciência e Tecnologia Agroindustrial, UFpel. Pelotas-RS. E-mail: ctajorge@ufpel.tche.br.

${ }^{6}$ Eng. Agr., Dr. Prof. Adjunto, UDESC - C. P. 1 281, CEP: 88520-000, Lages - SC.E-mail: leoruffato@yahoo.com.br

${ }^{7}$ Eng ${ }^{a}$ Agr.,Dra. Prof ${ }^{a}$ do Depto de Fitotecnia da UFPel, C. P. 354, 96010-900, Pelotas-RS; E-mail: derossiandrea@yahoo.com.br. 


\section{INTRODUÇÃO}

A Colômbia é o maior produtor mundial de frutos de Physalis peruviana L. (1753), seguida pela África do Sul (Chaves, 2006). No Brasil, o fruto é consumido como produto fino, com alto valor agregado, e está sendo incorporado nos cultivos de pequenas frutas. Seu plantio ainda é recente, mas está ampliando-se no Sul do País, principalmente nos municípios de Vacaria, Roca Sales e Áurea (Andrade, 2008).

O cultivo de physalis constitui-se em uma excelente alternativa para o mercado nacional e internacional. Isso se justifica pelo elevado conteúdo nutracêutico do fruto e pela possibilidade de incorporação da espécie nos cultivos orgânicos (Velasquez et al., 2007). Além disso, physalis pertence ao grupo dos frutos exóticos, desfrutando de alto destaque, caracterizado pelo consumo por grupos elites e pela distribuição exclusiva em hotéis, restaurantes e mercados especializados (Fischer \& Almanza, 1993).

A espécie pertence à família Solanaceae e caracteriza-se por apresentar cultivo bastante simples. A maior parte do manejo (tutoramento, adubação, aplicação de herbicidas e irrigação) ainda é feito semelhante ao utilizado para o cultivo do tomateiro. A planta é considerada arbustiva, perene e rústica, podendo atingir dois metros de altura. As folhas são aveludadas e triangulares, enquanto o talo principal, herbáceo e piloso, apresenta-se composto por 8 a 12 nós. $\mathrm{O}$ fruto constitui-se em uma baga carnosa, em forma de globo, com diâmetro que oscila entre 1,25 e 2,50 $\mathrm{cm}$ e peso entre 4 e $10 \mathrm{~g}$ (Chaves, 2006). Cada planta produz aproximadamente $2 \mathrm{~kg}$ de fruto por safra.

A physalis, por se tratar de um arbusto que pode formar uma ramificação muito densa, e cujos ramos são decumbentes, requer sistema de suporte. A condução é obrigatória, e o espaçamento adotado deve ser de acordo com o sistema de condução empregado. $\mathrm{O}$ tipo de condução e o amarrio requeridos são em função da densidade de semeadura, da topografia do terreno, da disponibilidade de materiais e seus custos (Sanabria \& Casella, 2002).

Diversos sistemas de condução podem ser empregados no cultivo de physalis, podendo ser utilizados os descritos em outras produções frutícolas ou, ainda, os empregados em cultivos de solanáceas, como no caso das utilizadas no tomateiro (Rufato et al., 2008). Os sistemas de condução frequentemente utilizados pelos agricultores brasileiros no cultivo de tomate é o "V" invertido, sistema triangular, sistema vertical com bambu e sistema vertical com fitilho ou tutoramento com fita plástica (Fontes \& Silva,
2002). Estes sistemas apresentam a característica geral de integrar práticas culturais e possibilitar ganhos quantitativos e/ou qualitativos e, ainda, são facilmente executáveis e já estão incorporados no conhecimento agrícola dos produtores (Sediyama et al., 2003).

Em relação ao fruto, este apresenta coloração alaranjada quando maduro e desenvolve-se dentro de um cálice formado por cinco sépalas. $O$ cálice protege o fruto contra insetos, pássaros, patógenos e condições climáticas adversas, e serve de fonte de carboidratos durante os primeiros 20 dias de crescimento. Além de prolongar a vida pós-colheita dos frutos em $2 / 3$, o cálice é considerado um indicador a ser observado na determinação do ponto de colheita (Ávila et al., 2006).

Os frutos de physalis, depois de colhidos, são classificados de acordo com a coloração da epiderme, em seis classes: $0=$ verde; 1 = amarelo-alaranjado; 2 = laranja-claro; 3 = laranja; 4 = laranja-escuro e 5 = laranja avermelhado, em que normalmente as três últimas fases são as que apresentam melhores características físico-químicas (Fischer \& Martinez, 1999). No entanto, a coloração do fruto não é o indicador utilizado durante a colheita, já que o cálice se encontra completamente fechado, impedindo a visualização da cor, o que gera dúvidas no reconhecimento do momento ideal de colheita (Andrade, 2008).

$\mathrm{O}$ objetivo neste trabalho foi avaliar as características físico-químicas dos frutos de physalis em função dos diferentes sistemas de condução e colorações do cálice utilizados.

\section{MATERIAL E MÉTODOS}

O experimento foi conduzido em 2008, no Laboratório de Bromatologia do Departamento de Ciência e Tecnologia Agroindustrial da Faculdade de Agronomia Eliseu Maciel-RS. Foram utilizados frutos de physalis (P. peruviana) provenientes da coleção pertencente ao Centro Agropecuário da Palma da Universidade Federal de Pelotas, localizado no Município do Capão do Leão-RS, latitude 31 ${ }^{\circ} 52^{\prime} 00^{\prime}$ " $\mathrm{S}$, longitude $52^{\circ} 21^{\prime} 24^{\prime \prime} \mathrm{W}$ e altitude 13,24 metros. O clima da região caracteriza-se como temperado úmido com verões quentes, conforme a classificação de Köeppen, do tipo "Cfa". A região possui temperatura e precipitação médias anuais de $17,9^{\circ} \mathrm{C}$ e $1.500 \mathrm{~m}$, respectivamente (EAPel, 2009).

A coleção empregada nesta pesquisa é formada por 45 plantas de $P$. peruviana, com idade de 10 meses, conduzidas nos sistemas " $\mathrm{V}$ " invertido e triangular, espaçadas $1,0 \times 0,5 \mathrm{~m}$ entre e dentro 
das fileiras, respectivamente, e 3,5 m entre linhas (Figura 1).

O solo onde se encontra a coleção de physalis, pertence à unidade de mapeamento Camaquã, sendo moderadamente profundo, com textura média no horizonte A e argilosa no B, classificados como Argissolo Vermelho-Amarelo. A área possui relevo plano, com cobertura vegetal predominante de Bidens pilosa L. e Cyperus rotundus L. (Rossi et al., 2007).

As plantas pertencentes à coleção são oriundas de sementes. As mudas foram produzidas em bandejas de isopor de 128 células preenchidas com substrato comercial, em condições de telado. O transplante foi realizado a campo, quando as plantas apresentavam aproximadamente $20 \mathrm{~m}$ de comprimento. Este foi realizado depois da correção do solo quanto ao pH e adubação de acordo com análise do solo. A recomendação de adubação foi de acordo com a cultura do tomateiro para uma expectativa de produção de $20 \mathrm{t} \mathrm{ha}^{-1}$.

Os frutos de physalis foram colhidos manual e aleatoriamente em diversas posições e orientações na planta, com utilização de luvas e caixas plásticas de colheita, lavadas e desinfetadas. A colheita foi realizada em função das diferentes colorações do cálice, sendo atribuídas cinco fases de colheita: 1= verde; 2 = verde-amarelado; 3 =amarelo-esverdeado; 4=amarelo, e 5=amarelo-amarronzado (Figura 2).

As fases de colheita foram determinadas com duas leituras, em lados opostos, na região equatorial do cálice, com o emprego do colorímetro Minolta CR300, fonte de luz D65 e $8 \mathrm{~m}$ de abertura, no padrão CIE-Lab. Nesse sistema, a coordenada L* expressa o grau de luminosidade da cor medida $\left(\mathrm{L}^{*}=0\right.$, preto a 100 , branco). O valor de $a^{*}$ expressa o grau de variação entre o vermelho e o verde $\left(a^{*}\right.$ negativo $=$ verde; $a^{*}$ positivo $=$ vermelho) e a coordenada $b^{*}, o$ grau de variação entre o azul e o amarelo ( $b^{*}$ negativo $=$ azul; $b^{*}$ positivo $=$ amarelo) (Quadro 1$)$.

Os valores $a^{*} \mathrm{e} b *$ foram usados para calcular o ângulo Hue ou matiz $\left(h^{\circ}=\tan ^{-1} \mathrm{~b}^{*} \cdot \mathrm{a}^{*-1}\right)$. Este ângulo caracteriza a cor exata do cálice no momento da colheita, conforme observado pela relação entre a Tabela 1 e Figura 2. A fase de coloração do cálice verde (1) (Figura 2) é comprovada pelo ângulo $\left(h^{\circ}\right)$ de 125.31 (Tabela 1 ), pois encontra-se próximo à faixa angular correspondente ao verde $\left(180^{\circ}\right)$. Outro extremo utilizado foi a fase de coloração do cálice amarelo (4) (Figura 2), que é comprovada pelo ângulo $\left(h^{\circ}\right)$ de 91.52 (Tabela 1), e encontrando-se muito próximo à faixa do ângulo amarelo $\left(90^{\circ}\right)$. As demais fases de coloração do cálice seguiram a mesma classificação descrita acima.

As características físico-químicas avaliadas foram: coloração da epiderme do fruto com o emprego do colorímetro Minolta CR - 300; massa do fruto, do cálice e total (fruto, cálice e talo), verificadas em balança digital, com resultados expressos em gramas por fruto g; diâmetro do fruto, através de duas leituras na região equatorial dos frutos com paquímetro digital, e os resultados foram expressos em milímetros (mm); firmeza dos frutos, em equipamento TA.XT plus equipado com microcomputador, realizando-se perfurações de $10 \mathrm{~m}$ na região equatorial dos frutos, usando ponteira de $2 \mathrm{~m}$ e velocidade de $1,0 \mathrm{~mm} . \mathrm{s}^{-1}$, com resultados expressos em Newton $(\mathrm{N})$; sólidos solúveis totais (SST), usando refratômetro de mesa Shimadzu, com correção de temperatura para $20^{\circ} \mathrm{C}$, utilizando-se de uma gota de suco puro de cada repetição e expressando resultado em ${ }^{\circ}$ Brix; acidez total titulável (ATT), avaliada por titulometria de neutralização, com diluição de $10 \mathrm{~mL}$ de suco puro em $90 \mathrm{~mL}$ de água destilada e titulação com solução de $\mathrm{NaOH} 0,1 \mathrm{~N}$ até que o suco atingisse pH 8,1, expressando-se o resultado em \% de ácido cítrico; razão (ratio) SST/ATT; e pH, determinado diretamente no suco dos frutos com o uso de um medidor de pH Digimed DMPH - 2, com correção automática de temperatura.

$\mathrm{O}$ delineamento experimental foi o inteiramente casualizado, com esquema fatorial $5 \times 2$ (colorações do cálice $\mathrm{x}$ sistemas de condução). A unidade experimental foi composta de dez frutos, sendo cada tratamento repetido três vezes. Os dados foram submetidos à análise de variância (ANOVA), aplicando o teste de Tukey, ao nível de 5\% de probabilidade de erro, para comparação das médias. As análises estatísticas dos resultados obtidos foram realizadas com programa estatístico Winstat 2.0 (Machado \& Conceição, 2003).

\section{RESULTADOS E DISCUSSÃO}

Observou-se interação significativa entre os fatores para as características químicas analisadas. Para os parâmetros físicos, no entanto, somente a variável independente colorações do cálice foi significativa.

Sólidos solúveis totais: as maiores médias foram obtidas na associação do sistema de condução triangular com a fase 4 , coloração amarela do cálice (Tabela 2). Os sistemas de condução proporcionaram aos frutos SST com médias semelhantes, com exceção da fase de coloração verde do cálice, onde o sistema "V" invertido apresentou menor SST. Portanto, a colheita manual poderia ser realizada a partir da fase 3 de coloração do cálice, independentemente do sistema de condução empregado, 
pois, segundo o CODEX (2005), frutos de physalis devem apresentar, no mínimo, $14{ }^{\circ}$ Brix para serem comercializados.

De acordo com Cardoso et al. (2005), o tipo de condução de plantas utilizado pode alterar a distribuição da radiação solar e a ventilação em torno das plantas. Assim, o sistema de tutoramento triangular proporcionaria maior luminosidade, estimulando a atividade fotossintética da planta e a produção de açúcares, os quais são armazenados nos frutos. Neste trabalho, não foram observadas diferenças significativas em SST, com exceção da fase de coloração verde do cálice, quando comparados os sistemas de condução "V" invertido e triangular.

A maior acidez total titulável foi obtida na fase 1 de coloração do cálice, combinada com o sistema de condução "V" invertido (Tabela 2). Os menores valores foram encontrados nas fases $4 \mathrm{e}$ 5 de coloração do cálice, para os dois sistemas de tutoramento aplicados. Esses resultados sugerem que o fruto, na fase 1, ainda não está no ponto de colheita, pois, de acordo com Lanchero et al. (2007), a acidez titulável decresce com o avanço do processo de maturação, em virtude do consumo dos ácidos orgânicos como substratos durante a respiração. Segundo Fischer et al. (2005), a forma de condução não possui influência direta na percentagem de ácido cítrico.

A razão (SST/ATT) fornece uma indicação de qualidade/maturação do fruto. Desse modo, quando se obtém uma razão muito baixa, há indicação de que se trate de um fruto mais ácido do que o desejável. Quando se observa um elevado valor, está-se diante de um fruto em estado de maturação mais avançado. Tais condições influenciam diretamente no sabor dos frutos de solanáceas (Borguini, 2002). As maiores médias foram encontradas quando o cálice apresentava coloração amarronzada (fase 5), associada ao sistema de tutoramento triangular (Tabela 2). Nesta mesma fase de coloração do cálice, podem-se observar os maiores valores no sistema "V" invertido. Contudo, os demais resultados desse sistema foram superiores aos encontrados no tutoramento triangular.

Segundo as normas da ICOTEC NTC 4580 (1999) para comercialização de physalis, a razão $\mathrm{SST} / \mathrm{ATT}$ (ratio) $\geq 6,0$ corresponde ao índice de maturação mínimo permitido para comercialização. Assim, os frutos colhidos em todas as fases de coloração do cálice utilizadas neste experimento poderiam ser comercializados. No entanto, esta variável deve estar associada a outras características de qualidade, como o conteúdo de sólidos solúveis totais, em que o valor mínimo deve ser de $14{ }^{\circ}$ Brix.
Em relação ao pH, a combinação do sistema de condução triangular com cálices de coloração amarelo-esverdeada, fase 3 , resultou em médias elevadas (Tabela 2). Entretanto, os menores resultados foram obtidos nas fases 1 e 2 de coloração do cálice associadas ao mesmo sistema de tutoramento. Acredita-se que a coloração do cálice exerça maior influência nas respostas do fator $\mathrm{pH}$ do que o sistema de condução utilizado (Fischer et al., 2005). Para Ferreira (2004), o $\mathrm{pH}$ decresce significativamente com os primeiros sinais de maturação nos frutos e aumenta levemente com o estádio de passado. Conforme Novoa et al. (2006), o pH é um parâmetro importante na regulação do metabolismo. Nos frutos, mais de $90 \%$ do volume celular são ocupados pelo vacúolo, que é muito ácido e que apresenta $\mathrm{pH}$ inferior a 5, valor que coincide com os obtidos neste trabalho. De acordo com Lanchero et al. (2007), o fator $\mathrm{pH}$ é considerado variável pouco eficiente na colheita de physalis.

A coloração da epiderme dos frutos não apresentou interação com fatores avaliados neste trabalho, mas a variável independente coloração do cálice foi significativa. As médias mais acentuadas para o ângulo Hue e para a coordenada $\mathrm{a}^{*}$ foram obtidas na fase de coloração do cálice 1 e 5 , respectivamente (Tabela 3). Tais informações indicam que os frutos apresentavam coloração mais verde (maior Hue) na fase 1, e que na fase 5 estavam laranja-avermelhados (maior $\mathrm{a}^{*}$ ). Para as variáveis luminosidade e coordenada $b *$ foi verificado, na fase 5 , frutos com amarelo mais brilhante (maior luminosidade) e, na fase 1 , as menores intensidades de amarelo (menor $b^{*}$ ). Segundo as normas da ICOTEC NTC 4580 (1999), os frutos de physalis devem ser comercializados quando a epiderme apresentar coloração laranja. Portanto, a colheita poderia ser realizada a partir da fase 3 de coloração do cálice.

Para a variável massa dos frutos, o maior resultado foi obtido na fase 5 de coloração do cálice, quando se apresentavam amarelo-amarronzados, entretanto esta só diferiu da fase 1 (Tabela 4). Entre as fases 2 e 5 de coloração, a massa dos frutos variou de 8,85 a 10,22 g, estando dentro da faixa de valores de massa de frutos encontrados por Fischer \& Almanza (1993), a qual foi de 4 a $10 \mathrm{~g}$. A massa dos frutos é considerada uma importante característica, pois está relacionada linearmente com o grau de desenvolvimento e/ou de amadurecimento, exceto quando os frutos se encontram em estado avançado de maturação (Costa et al., 2004).

Quando verificada a massa do cálice, a resposta foi contrária àquela obtida para a massa dos frutos. A maior média ocorreu nos cálices de coloração verde, fase 1 (Tabela 4). Para esta variável, observou-se redu- 
ção na massa à medida que houve evolução nas fases de coloração do cálice. As respostas obtidas podem ser elucidadas, pois o cálice, durante os primeiros 20 dias de crescimento dos frutos, serve como fonte de carboidratos, além de apresentar, nesta mesma fase, maior retenção de água e conteúdo de clorofilas, justificando a massa do cálice mais elevada quando estes apresentam coloração verde (Ávila et al., 2006).

$\mathrm{Na}$ fase 4 de coloração do cálice, foram observados os maiores valores de massa total (Tabela 4). No entanto, estes resultados diferiram apenas dos verificados na fase 1 , coloração verde do cálice. A fase 5 apresentou médias de massa total intermediárias, representando as proporções de massa adequadas entre cálice, fruto e talo. Estas informações estão de acordo com as obtidas por Fischer \& Martínez (1999), que em seu estudo mencionam que frutos de physalis são comercializados em conjunto, com talo e cálice. Desse modo, para se obter resposta de massa total equivalente ao praticado no comércio, devem-se mensurar os três componentes.

$\mathrm{Na}$ avaliação do diâmetro dos frutos, a maior média foi obtida na fase 5 de coloração do cálice, porém esta diferiu significativamente apenas das obtidas em frutos na fase 1 (Tabela 4). Conforme Ávila et al. (2006), os frutos no estádio de maturação verde apresentam valores de diâmetro inferiores aos demais. Isto ocorre, na maioria das vezes, porque os frutos verdes ainda não atingiram seu completo desenvolvimento fisiológico.

Na coloração verde do cálice, (fase 1), foram observadas as maiores médias de firmeza, sem diferir, no entanto, das médias obtidas em cálices de coloração verde-amarelada da fase 2 (Tabela 4). Foi verificada redução de firmeza à medida que avançavam as fases de coloração do cálice. Segundo Velasques et al. (2007), a firmeza é o melhor indicador prático da maturação de um fruto, pois esta avaliação determina os níveis ótimos para consumo, transporte e manejo. Esta característica, no entanto, pode ser utilizada somente quando o fruto é comercializado sem o cálice. Porém, a maioria dos produtores comercializa com o cálice; logo, tal avaliação prejudicaria o comércio da fruta, pois seria necessária a retirada do cálice, qual responsável por agregar valor.

Quadro 1 - Descrição da leitura do colorímetro Minolta CR-300, fonte de luz D65 e 8 mm de abertura, no padrão CIE-Lab. FAEM/UFPel, Capão do Leão-RS, 2008.

\begin{tabular}{|l|l|}
\hline \multicolumn{1}{|c|}{ Coordenada } & \multicolumn{1}{c|}{ Grau de variação } \\
\hline L- Luminosidade & $\mathrm{L}=0=$ preto, $\mathrm{L}=100=$ branco \\
a- variação entre o vermelho e o verde & $-\mathrm{a}=$ verde, $+\mathrm{a}=$ vermelho \\
b- variação entre o azul e o amarelo & $-\mathrm{b}=\mathrm{azul},+\mathrm{b}=$ amarelo \\
$h^{\text {o }}$ ângulo Hue ou matiz & $h^{\mathrm{o}}=\operatorname{tang}^{-1} \mathrm{~b} \cdot \mathrm{a}^{-1}$ \\
\hline
\end{tabular}

TABELA 1- Descrição das fases de coloração do cálice de frutos de physalis no momento da colheita em função de L*, a*, b* e h ${ }^{\circ}$. FAEM/UFPel, Capão do Leão-RS, 2008.

\begin{tabular}{ccccc}
\hline Fases de coloração do cálice & $\mathrm{L}^{*}$ & $\mathrm{a}^{*}$ & $\mathrm{~b}^{*}$ & $h^{\circ}$ \\
\hline Verde (1) & 49,98 & $-7,40$ & 15,71 & 125,31 \\
Verde-amarelado (2) & 53,61 & $-6,54$ & 20,71 & 117,76 \\
Amarelo-esverdeado (3) & 56,22 & 0,01 & 20,66 & 109,56 \\
Amarelo (4) & 57,14 & 12,35 & 14,47 & 91,52 \\
Amarelo-amarronzado (5) & 54,99 & 2,61 & 20,06 & 79,28 \\
\hline
\end{tabular}

$\mathrm{L}^{*}(0=$ preto, $100=$ branco $) ; \mathrm{a}^{*}(+\mathrm{a}=$ vermelho, $-\mathrm{a}=$ verde $) ; \mathrm{b}^{*}(+\mathrm{b}=$ amarelo, $-\mathrm{b}=\mathrm{azul}) ;$ ângulo $\mathrm{h}^{\mathrm{o}}\left(0^{\circ}=\right.$ vermelho, $90^{\circ}=$ amarelo, $180^{\circ}=$ verde, $360^{\circ}=$ azul $)$. 
TABELA 2 - Sólidos solúveis totais (SST), acidez total titulável (ATT), pH, SST/ATT em frutos de physalis, no momento da colheita, em cinco fases de coloração do cálice e em dois sistemas de condução. FAEM/UFPel, Capão do Leão-RS, 2008.

\begin{tabular}{|c|c|c|c|}
\hline \multirow{2}{*}{ Variáveis } & \multirow{2}{*}{ Fases de coloração do cálice } & \multicolumn{2}{|c|}{ Sistemas de condução } \\
\hline & & "V" invertido & Triangular \\
\hline \multirow{5}{*}{$\mathrm{SST}\left({ }^{\circ}\right.$ Brix $)$} & Verde (1) & $10,86 \mathrm{~b} \quad \mathrm{~B}$ & $11,43 \mathrm{~b}$ A \\
\hline & Verde-amarelado (2) & $13,46 \mathrm{ab} A$ & $13,50 \mathrm{ab} A$ \\
\hline & Amarelo-esverdeado (3) & $14,36 \mathrm{a} \quad \mathrm{A}$ & $14,60 \mathrm{a} \quad \mathrm{A}$ \\
\hline & Amarelo (4) & $14,43 \mathrm{a} \quad \mathrm{A}$ & $15,30 \mathrm{a} \quad \mathrm{A}$ \\
\hline & Amarelo- amarronzado (5) & 14,53 a $\mathrm{A}$ & 14,43 a $\mathrm{A}$ \\
\hline \multirow{5}{*}{ ATT (\% ác. cítrico) } & Verde (1) & 0,89 a $\mathrm{A}$ & $0,82 \mathrm{a} \quad \mathrm{B}$ \\
\hline & Verde-amarelado (2) & $0,74 \mathrm{~b} \quad \mathrm{~B}$ & $0,79 \mathrm{ab} A$ \\
\hline & Amarelo-esverdeado (3) & 0,76 bc $\mathrm{A}$ & $0,74 \mathrm{~b} \quad \mathrm{~B}$ \\
\hline & Amarelo (4) & 0,69 c $A$ & $0,69 \mathrm{~b} \quad \mathrm{~A}$ \\
\hline & Amarelo- amarronzado (5) & 0,66 c A & 0,54 c $B$ \\
\hline \multirow{5}{*}{$\begin{array}{c}\text { Relação SST/ATT } \\
(\text { Ratio })\end{array}$} & Verde (1) & $14,34 \mathrm{~d} \mathrm{~A}$ & 13,98 eA \\
\hline & Verde-amarelado (2) & 19,51 bc A & $18,11 \mathrm{~cd} \mathrm{~B}$ \\
\hline & Amarelo-esverdeado (3) & $21,76 \mathrm{ab} A$ & 21,17 bc A \\
\hline & Amarelo (4) & $18,73 \mathrm{cdA}$ & $16,26 \mathrm{deB}$ \\
\hline & Amarelo- amarronzado (5) & $22,18 \mathrm{a} \quad \mathrm{B}$ & $26,60 \mathrm{a} \quad \mathrm{A}$ \\
\hline \multirow{5}{*}{$\mathrm{pH}$} & Verde (1) & 3,52 b A & 3,41 c $B$ \\
\hline & Verde-amarelado (2) & $3,53 \mathrm{~b} \quad \mathrm{~A}$ & 3,43 c $B$ \\
\hline & Amarelo-esverdeado (3) & 3,48 c $B$ & 3,85 a $\mathrm{A}$ \\
\hline & Amarelo (4) & 3,49 c A & 3,49 bc $\mathrm{A}$ \\
\hline & Amarelo- amarronzado (5) & $3,61 \mathrm{ab} \mathrm{A}$ & $3,58 \mathrm{~b} \quad \mathrm{~A}$ \\
\hline
\end{tabular}

TABELA 3 - Valores de luminosidade (L), coordenadas $a^{*}, b^{*}$ e ângulo Hue $\left(h^{\circ}\right)$ da epiderme dos frutos de physalis em função de cinco fases de coloração do cálice, no momento da colheita. FAEM/ UFPel, Capão do Leão-RS, 2008.

\begin{tabular}{lcccc}
\hline \multirow{2}{*}{ Fases coloração do cálice } & \multicolumn{4}{c}{ Epiderme da Fruta } \\
\cline { 2 - 5 } & $\mathrm{L}^{*}$ & $\mathrm{a}^{*}$ & $\mathrm{~b}^{*}$ & $h^{\circ}$ \\
\hline Verde (1) & $62,19 \mathrm{a}$ & $-4,47 \mathrm{~b}$ & $29,28 \mathrm{a}$ & $105,06 \mathrm{a}$ \\
Verde-amarelado (2) & $62,90 \mathrm{a}$ & $7,47 \mathrm{a}$ & $35,39 \mathrm{a}$ & $78,19 \mathrm{~b}$ \\
Amarelo-esverdeado (3) & $66,49 \mathrm{a}$ & $7,79 \mathrm{a}$ & $36,75 \mathrm{a}$ & $77,61 \mathrm{~b}$ \\
Amarelo (4) & $63,05 \mathrm{a}$ & $8,01 \mathrm{a}$ & $32,34 \mathrm{a}$ & $75,17 \mathrm{~b}$ \\
Amarelo-amarronzado (5) & $67,04 \mathrm{a}$ & $8,83 \mathrm{a}$ & $38,45 \mathrm{a}$ & $74,69 \mathrm{~b}$ \\
\hline
\end{tabular}

*Médias seguidas pela mesma letra minúscula na coluna não diferem entre si, pelo teste de Tukey $(\mathrm{p} \leq 0,05)$. $\mathrm{L}^{*}(0=$ preto, $100=$ branco $) ; \mathrm{a}^{*}(+\mathrm{a}=$ vermelho, $-\mathrm{a}=$ verde $) ; \mathrm{b}^{*}(+\mathrm{b}=$ amarelo, $-\mathrm{b}=$ azul $) ;$ ângulo $\mathrm{h}^{\mathrm{o}}\left(0^{\circ}=\right.$ vermelho, $90^{\circ}=$ amarelo, $180^{\circ}=$ verde, $360^{\circ}=$ azul $)$. 
TABELA 4-Massa do fruto, do cálice e total ( $\mathrm{g}$ ), diâmetro $(\mathrm{m})$ e firmeza ( $\mathrm{N}$ ) em função das cinco colorações do cálice de frutos de physalis, no momento da colheita. FAEM/UFPel, Capão do Leão-RS, 2008.

\begin{tabular}{lccccc}
\hline \multicolumn{1}{c}{$\begin{array}{c}\text { Fases de coloração do } \\
\text { cálice }\end{array}$} & $\begin{array}{c}\text { Massa de fruto } \\
(\mathrm{g})\end{array}$ & $\begin{array}{c}\text { Massa do cálice } \\
(\mathrm{g})\end{array}$ & $\begin{array}{c}\text { Massa total } \\
(\mathrm{g})\end{array}$ & $\begin{array}{c}\text { Diâmetro } \\
(\mathrm{m})\end{array}$ & \multicolumn{2}{c}{ Firmeza } \\
$(\mathrm{N})$
\end{tabular}

*Médias seguidas pela mesma letra minúscula na coluna não diferem entre si, pelo teste de Tukey $(\mathrm{p} \leq 0,05)$.
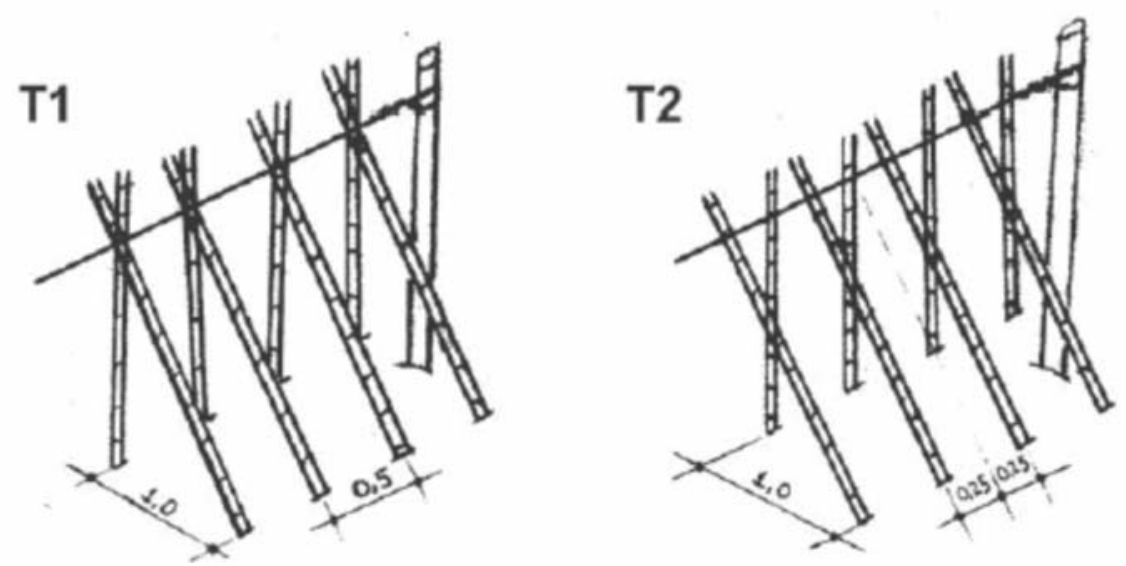

Fonte: Marin et al. (2005).

FIGURA 1- Esquema ilustrativo do sistema de condução de plantas de physalis. FAEM/UFPel, Capão do Leão-RS, 2008. * (T1) "V”invertido e (T2) triangular.

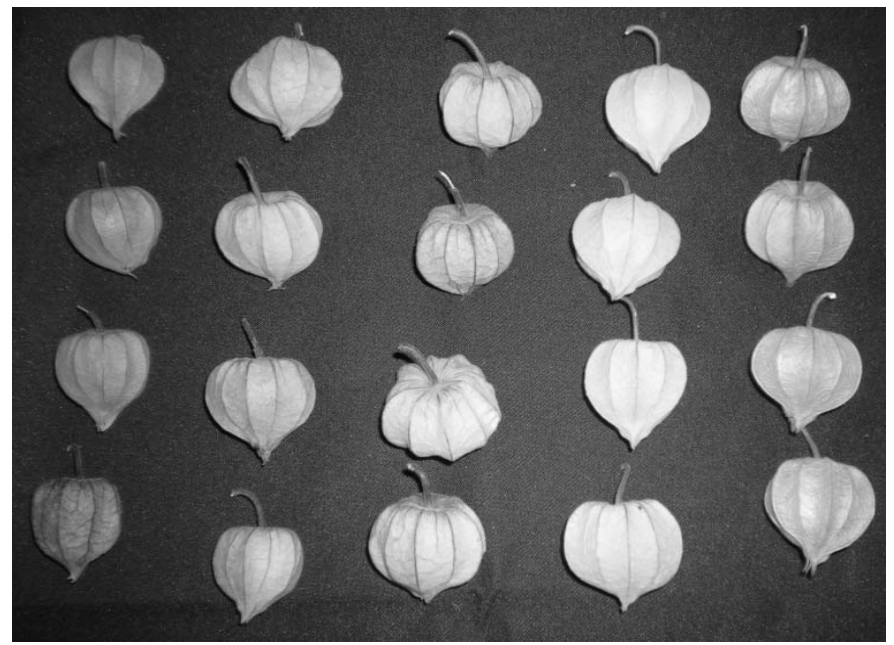

Fonte: LIMA, C.S.M. (2008)

FIGURA 2 - Fases de coloração do cálice de frutos de physalis. FAEM/UFPel, Capão do Leão-RS, 2008. Da esquerda para direita: (1) verde, (2) verde-amarelado, (3) amarelo-esverdeado, (4) amarelo e (5) amarelo-amarronzado. 


\section{CONCLUSÕES}

1- A caracterização físico-química de frutos de physalis e sua associação com a coloração do cálice possibilitam a utilização do último como indicador do ponto de colheita adequado para os frutos.

2- Os sistemas de condução, "V" invertido e triangular, afetam as características químicas dos frutos de physalis.

3- A partir da fase três de coloração do cálice (amarelo-esverdeado), em ambos os sistemas de condução avaliados, "V" invertido e triangular, a colheita de physalis pode ser realizada, pois os frutos já apresentam os atributos mínimos de qualidade exigidos.

\section{AGRADECIMENTOS}

À Coordenação de Aperfeiçoamento de Pessoal de Nível Superior (CAPES) e ao Conselho Nacional de Desenvolvimento Científico e Tecnológico (CNPq), pelo apoio financeiro e pela concessão de bolsas aos pesquisadores deste projeto.

\section{REFERÊNCIAS}

ANDRADE, L. Physalis ou uchuva: Fruta da Colômbia chega ao Brasil. Revista Rural, São Paulo, s.v., n. 38, p. 11-12, 2008.

ÁVILA, A.J.,; MORENO, P.; FISCHER, G.; MIRANDA, D. Influencia de la madurez del fruto y del secado del cáliz en uchuva (Physalis peruviana L.), almacenada a $18^{\circ} \mathrm{C}$. Acta Agronómica Colombiana, Palmira, v. 55, n. 4, p. 29-38, 2006.

BORGUINI, R.G. Tomate (Lycopersicon esculentum Mill.) orgânico: o conteúdo nutricional e a opinião do consumidor. 2002. 127 f. Dissertação (Mestrado) - Escola Superior de Agricultura Luiz de Queiroz, Universidade de São Paulo, Piracicaba, 2002.

CARDOSO, S.C.; SOARES, A.C.F.; BRITO, A. dos S.; CARVALHO, L.A. de; PEIXOTO, C.C.; PEREIRA, M.E.C.; GOES, E. Qualidade de frutos de tomateiro com e sem enxertia. Bragantia, Campinas, v. 65 , n. 2, p. 269-274, 2005.
CHAVES, A.C. Propagação e avaliação fenológica de Physalis sp na região de Pelotas-RS. 2006. 65 f. Tese (Doutorado) - Universidade Federal de Pelotas, Pelotas, 2006.

CODEX. Norma del codex para la uchuva, México, 2005. (Codex Stan 226 2001)

COSTA, N.P. da; LUZ, T.L.B.; GONÇALVES, E.P.; BRUNO, R. de L. A. Caracterização físico-química de frutos de umbuzeiro (Spondias tuberosa Arr. Câm.), colhidos em quatro estádios de maturação. Bioscience Journal, Uberlândia, v. 20, n. 2, p. 6571, 2004.

EAPel. Estação Agroclimatólogica de Pelotas. Disponível em: $<$ http://www.cpact.embrapa.br/agromet/ estacao/index.html > Acesso em: 20 jan. 2009.

FERREIRA, S.M.R. Características de qualidade do tomate de mesa (Lycopersicon esculentum Mill.) cultivado nos sistemas convencional e orgânico comercializado na região metropolitana de Curitiba. 2004. 249 f. Tese (Doutorado) - Universidade Federal do Paraná, Curitiba, 2004.

FISCHER, G.; ALMANZA, P.J. Nuevas tecnologías en el cultivo de la uchuva Physalis peruviana L. Agrodesarrollo, Tunja, v. 4, n. 1-2, p. 294, 1993.

FISCHER, G.; MARTÍNEZ; O. Calidad y madurez de la uchuva (Physalis peruviana L.) emrelación con la coloracióndel fruto. Agronomía Colombiana, Bogotá, v.16, n. 1-3, p. 35-39, 1999.

FISCHER, G.; MIRANDA, D.; PIEDRAHITA, W.; ROMERO, J. Avances en cultivo, poscosecha y exportación de la uchuva (Physalis peruviana L.) en Colombia. Bogotá: Universidad Nacional de Colômbia, 2005. 221 p.

FONTES, P.C.R.; SILVA, D.J.H. Produção de tomate de mesa. Viçosa: Aprenda Fácil, 2002. 193 p.

ICONTEC. Frutas frescas: uchuva, especificaciones. Bogotá: Instituto Colombiano de Normas Técnicas, 1999. 15 p. (Norma Técnica Colombiana NTC, 4580). 
LANCHERO, O.; VELANDIA, G.; FISCHER, G.; VARELA, N.C.; GARCÍA, H. Comportamiento de la uchuva (Physalis peruviana L.) en poscosecha bajo condiciones de atmosfera modificada activa. Revista Corpoica - Ciencia y Tecnología Agropecuaria, Bogotá, v.8, n.1, p. 61-68, 2007.

MACHADO, A.A.; CONCEIÇÃO, A.R. Sistema de análise estatística para windows. WinStat: versão 2.0. Pelotas: UFPel, 2003.

MARIN, B.G.; SILVA, D.J.H.; GUIMARÃES, M.A.; BELFORT, G. Sistemas de tutoramento e condução do tomateiro visando a produção de frutos para consumo in natura. Horticultura Brasileira, Brasília, v. 23, p. 951-955. 2005.

NOVOA, R., M.; BOJACÁ, J.; GALVIS, Y.; G. FISCHER. La madurez del fruto y el secado Del cáliz influyen en el comportamiento poscosecha de la uchuva (Physalis peruviana L.) almacenada. Agronomía Colombiana, Bogotá, v. 24, n. 1, p. 77-86, 2006.

RUFATO, L.; RUFATO, A.R.; SCHELEMPER,C.; LIMA,C.S.M.; KRETZSCHMAR, A. A.A. Aspectos técnicos da cultura da physalis. Lages: CAV/ UDESC; Pelotas: UFPel, 2008. 100p.
ROSSI, A. de ; RUFATO, L. ; GIACOBBO, C. L.; COSTA, V.B.; VITTI, M. R.; MENDEZ.; M. E. G.;FACHINELLO, J. C. Diferentes manejos da cobertura vegetal de aveia-preta em pomar no Sul do Brasil. Bragantia, Campinas, v.66, n.3, p.457463, 2007.

SANABRIA, V.M.; CASELLA, E.C. Estudio de caso cultivo de Uchuva (Physalis peruviana L.). 2002. 37 f. Monografia (Trabalho de Graduação em Agronomia) - Facultad de Agronomía, Universidad Nacional de Colômbia, Bogotá, 2002.

SEDIYAMA, M.A.N.; FONTES, P.C.R.; SILVA, D.J.H. Práticas culturais adequadas ao tomateiro. Informe Agropecuário, Belo Horizonte, v. 24, n.219, p.19-25. 2003.

VELASQUEZ, H.J.C.; GIRALDO, O.H.B.; ARANGO, S.S.P. Estudio preliminar de la resistencia mecánica a la fractura y fuerza de firmeza para frut fruta de uchuva ( Physalis peruviana L.). Revista Facultad Nacional de Agronomía, Medellín, v. 60, n. 1, p. 3785-3796, 2007. 